\title{
The role of maintenance pemetrexed in the treatment of non-small-cell lung cancer
}

\author{
This article was published in the following Dove Press journal: \\ Lung Cancer:Targets and Therapy \\ 29 July 2010 \\ Number of times this article has been viewed
}

\author{
Saeed Rafii \\ Michael H Cullen \\ Department of Medical Oncology, \\ Queen Elizabeth Hospital, University \\ Hospital Birmingham, Edgbaston, \\ Birmingham, BI 5 2TH, \\ United Kingdom
}

\begin{abstract}
Until recently, the weight of evidence has supported the discontinuation of chemotherapy in advanced non-small-cell lung cancer (NSCLC) after 4-6 cycles of induction therapy. This allows patients with limited life expectancy a "treatment holiday." A minority of cases then go on to receive second-line therapy, although many deteriorate rapidly and never receive further active treatment. There has been renewed interest in the concept of maintenance from trials with pemetrexed and erlotinib. Both these agents can be given for long periods without serious cumulative toxicity in most patients. Both trials have shown significant extension of progression free survival in placebo-controlled trials. In cases who are not receiving pemetrexed as induction therapy, a statistically significant 5-month prolongation of overall survival in nonsquamous NSCLC has been reported. Treatment was well tolerated. This effect may reflect the early administration of an active second-line agent and it remains to be seen whether similar benefits will accrue to patients having pemetrexed as induction therapy.
\end{abstract}

Keywords: inoperable NSCLC, chemotherapy, targeted treatments

\section{Treatment of advanced non-small-cell lung cancer}

The majority of patients with non-small-cell lung cancer (NSCLC) present with inoperable metastatic or advanced disease at the time of diagnosis. With the 5-year survival rate ranging from $8 \%$ to $15 \%$, the prognosis for many of the patients with advanced or metastatic NSCLC is poor. ${ }^{1}$ For patients with good performance status (PS), palliative systemic chemotherapy with platinum regimens offers modest life extension and improves quality of life. A meta-analysis in 1995 analyzed individual data from 9,387 patients in 52 clinical trials and confirmed the benefit of chemotherapy. ${ }^{2}$ The authors updated this meta-analysis in 2008 using data from 2,714 patients from 16 randomized clinical trials including newer chemotherapy regimens. ${ }^{3}$ Chemotherapy proved to reduce the risk of death by $13 \%$ (hazard ratio $[\mathrm{HR}]=0.87 ; P=0.005)^{2}$ and increase the survival by $9 \%$ at 12 months translating to absolute survival benefit of 1.5 months. $^{3}$

Adding a second active agent such as paclitaxel, docetaxel, gemcitabine, or vinorelbine to cisplatin has shown survival benefit over single-agent treatment, cisplatin. Addition of second agent proved to have superior response and survival rate over single-agent therapy in a meta-analysis conducted by Delbaldo et $\mathrm{al}^{4}$ in 2004 that included 13,601 patients from 65 trials.

Several platinum-based doublet therapies incorporating agents such as gemcitabine, docetaxel, paclitaxel, vinorelbine, or more recently pemetrexed for NSCLC of nonsquamous histology have become available. There have been several attempts
Correspondence: Dr. Saeed Rafii

Department of Medical Oncology, Queen Elizabeth Hospital, University Hospital Birmingham, Edgbaston, Birmingham, B I5 2TH, United Kingdom Tel +44 (0) I2I 6272446

Email s.rafii@gmail.com 
to compare different platinum-based 2 drug combinations. In a study by the South-West Oncology Group (SWOG), for instance, 408 patients were randomized to receive vinorelbine plus cisplatin or paclitaxel plus carboplatin. No significant difference was demonstrable (response rate, $28 \%$ vs $25 \%$, respectively; median survival, 8 months in both arms). ${ }^{5}$ In another phase 3 trial by the Eastern Cooperative Oncology (ECOG), 1,151 patients were randomized in 4 arms to assess the survival benefits between cisplatin plus paclitaxel, cisplatin plus gemcitabine, cisplatin plus docetaxel, or carboplatin plus paclitaxel. Again no difference in response or survival rate was observed. ${ }^{6}$

Neither of these trials was powered to show clinically relevant differences and little attention was paid to different outcomes by histology. Nevertheless, a general belief developed that a "plateau" of efficiency for chemotherapy had been reached. This belief has since shown to be mistaken.

The optimum number of treatment cycles for NSCLC has also been the subject of clinical trials. Von Plessen et $\mathrm{al}^{7}$ compared 3 vs 6 cycles of doublet therapy, and Socinski et $\mathrm{al}^{8}$ randomized patients to receive either 4 cycles of carboplatin plus paclitaxel or continue the same regimen until disease progression. The number of cycles was limited due to drug-related toxicities and in general, no benefit in overall survival (OS) was observed in patients who received more than 4 cycles. The American Society of Clinical Oncology (ASCO) guideline recommends that first-line treatment should be administered between 4 and 6 cycles. ${ }^{9}$

\section{Recent developments}

The discovery of targeted therapies such as epidermal growth factor receptor - tyrosine kinase inhibitors (EGFR-TKIs), gefitinib and erlotinib, is beginning to change the way we treat advanced NSCLC. Also interest in novel chemotherapy agents, particularly pemetrexed, has been stimulated by data showing its superiority in nonsquamous tumors and better tolerance in both second-line setting and first-line setting, as well as in remission maintenance.

\section{Pemetrexed}

Pemetrexed (Alimta ${ }^{\circledR}$; Eli Lilly and Company, Indianapolis, Indiana, USA) is a new generation, multitargeted antifolate drug. It has a very high affinity for folate receptors and enters the cell through reduced folate carrier like methotrexate. Other modes of entry into the cell include low $\mathrm{pH}$ transporter and endolysis by folate receptor- $\alpha .{ }^{10}$ Intracellularly, it is then polyglutamated to its active form pentaglutamide by folylpolyglutamate synthase.
The polyglutamated form of pemetrexed is a potent inhibitor of thymidilate synthase (TS), an enzyme needed for synthesis of thymidine nucleotides. ${ }^{11}$ Inhibition of TS stops transformation of deoxyuridine monophosphate to deoxythymidine monophosphate, resulting in decreased thymidine necessary for DNA synthesis and cell replication subsequently inhibiting cell growth. In addition to TS, other enzymes such as dihydrofolate reductase, aminoimidazole carboxamide ribonucleotide formyltransferase, and glycinamide ribonucleotide formyltransferase are inhibited by pemetrexed. ${ }^{12}$ The latter 2 are folate-dependent enzymes that are involved in the de novo biosynthesis of purine. The ability of pemetrexed to target multiple enzymes involved in both thymidine and hypoxanthine synthesis pathways accounts for its cytotoxicity and inhibition of cell proliferation.

\section{Pharmacokinetics}

Almost $80 \%$ of pemetrexed is bound to plasma proteins. It is rapidly eliminated from the plasma by urinary excretion with a half-life of 3.5 hours. Therefore, its plasma clearance decreases with reduced renal function resulting in increased systemic exposure. Pharmacokinetics of pemetrexed is not affected by elevated transaminases or total bilirubin due to its limited hepatic metabolism. ${ }^{13}$

\section{Pemetrexed as a second-line treatment of NSCLC}

Pemetrexed was first approved as a second-line treatment of NSCLC based on a trial by Hanna et $\mathrm{al}^{14}$ in 2004. In this trial, which is designed to compare the efficacy and toxicity of pemetrexed vs docetaxel, 571 patients with advanced NSCLC with PS 0-2 who had progressed following treatment with platinum-based combinations, were randomized to receive pemetrexed (283 patients) or docetaxel (288 patients). There was no significant difference in median progression free survival (PFS), median survival time, or overall response rates between pemetrexed and docetaxel $(9.1 \%$ and $8.8 \%$, respectively; $P=0.105)$. However, pemetrexed showed a better toxicity profile and less hospitalization when compared with docetaxel.

\section{Pemetrexed as a first-line treatment of NSCLC}

A trial by Scagliotti et a ${ }^{15}$ published in 2008 compared cisplatin plus pemetrexed with cisplatin plus gemcitabine as a first-line treatment of NSCLC. The end point of this phase 3 , randomized study was OS. A total of 1,725 
chemotherapy-naive patients with stage 3B or 4 NSCLC and an ECOG PS of 0-1 were randomized to receive either cisplatin on day 1 plus gemcitabine on days 1 and 8 ( 863 patients) or cisplatin and pemetrexed on day 1 (862 patients) for every 3 weeks for up to 6 cycles. There was no difference in OS between the 2 arms of the study (median survival, 10.3 vs 10.3 months, respectively; $\mathrm{HR}=0.94$; $95 \%$ confidence interval [CI], 0.84-1.05). However, the pemetrexed arm showed a better safety profile.

In addition, OS with the cisplatin plus pemetrexed combination was superior to cisplatin plus gemcitabine in patients with adenocarcinoma $(\mathrm{n}=847 ; 12.6$ vs 10.9 months, respectively; $\mathrm{HR}=0.84 ; 95 \% \mathrm{CI}, 0.71-0.99 ; P=0.03)$ and large-cell carcinoma $(\mathrm{n}=153 ; 10.4$ vs 6.7 months, respectively; $\mathrm{HR}=0.67 ; 95 \% \mathrm{CI}, 0.48-0.96 ; P=0.03)$. Conversely, patients with squamous histology had superior survival with cisplatin plus gemcitabine.

\section{Concept of early second-line treatment}

Survival advantages from agents such as docetaxel, pemetrexed, and erlotinib in the second-line setting have paved the way to a new approach to second-line treatment. Oncologists have started to look at the effectiveness of these drugs after completion of first-line treatment and before disease progression. In this approach, good PS patients with responsive or stable disease after first-line therapy are considered for continuation of treatment with an active drug other than those used for induction.

This approach allows more suitable patients to be exposed to an alternative active therapy than would be the case if one waited for disease progression since, in routine practice, the vast majority of NSCLC patients never receive second-line therapy. This is because rapid deterioration, poor PS, and comorbidities intervene.

\section{Maintenance therapy, definitions, and strategies}

Based on the above rationale, the concept of maintenance therapy in NSCLC has been formed. There is no consensus, however, on the definition of maintenance, and other terms such as consolidation and early second-line treatment have also been used interchangeably. Furthermore, different strategies, including continuing chemotherapy (1) using 1 or more agents given in induction chemotherapy; (2) using a chemotherapy agent not used in first-line therapy (noncross resistant drug); or (3) using targeted therapies have been employed until disease progression.
In a phase 3 randomized trial, Fidias et $\mathrm{al}^{16}$ studied the efficacy and safety of immediate vs delayed docetaxel after first-line treatment. Following treatment with gemcitabine plus carboplatin, 398 patients with advanced, nonprogressive NSCLC were randomized to receive immediate docetaxel or at the point of disease progression. Patients in the immediate-docetaxel arm had statistically significant prolongation of median PFS compared with the 156 patients in the delayed-docetaxel arm (5.7 vs 2.7 months, respectively; $P=0.0001$ ). Although the median OS for immediate docetaxel (12.3 months) was greater than that for delayed docetaxel ( 9.7 months), the difference was not statistically significant $(P=0.0853)$. One criticism of this study was that the follow-up intervals for patients in the control arm were long (every 3 months) and only $24 \%$ of controls were eligible to receive second-line treatment.

\section{Maintenance pemetrexed}

A multicenter randomized, double blind, placebo-controlled phase 3 trial (JMEN) evaluated pemetrexed as a maintenance therapy. ${ }^{17}$ Patients with stage 3B or 4 NSCLC who had nonprogressive disease after first-line treatment with a nonpemetrexed-containing platinum doublet were selected for this study. All 663 patients had PS 0-1 and were randomized to receive pemetrexed $500 \mathrm{mg} / \mathrm{m}^{2}$ plus best supportive care (BSC) or placebo plus BSC for every 3 weeks. The trial was designed as a superiority study and patients were randomized in a 2:1 ratio with 441 patients in the pemetrexed arm and 222 patients in the placebo arm. PFS was set as the primary end point. Median PFS was significantly higher in the pemetrexed group than in the placebo (4.3 vs 2.6 months, respectively; $P<0.00001)$.

A subgroup of 481 patients in the study had nonsquamous non-small cell histology. Of this subgroup, 325 patients received pemetrexed and 156 placebo. Patients in the nonsquamous subgroup had improved PFS with pemetrexed (4.7 vs 2.6 months; $\mathrm{HR}=0.47 ; P<0.00001$ ). No difference in median PFS was observed between the 2 arms in patients with squamous histology (2.4 vs 2.5 months; $\mathrm{HR}=1.03$; $P=0.896)$.

Pemetrexed increased median OS when compared with placebo (13.4 vs 10.6 months; HR $=0.79 ; P=0.012$ ). However, the OS advantage with pemetrexed was far greater in patients with nonsquamous (15.5 vs 10.3 months; $\mathrm{HR}=0.70 ; P=0.002)$ than in those with squamous histology (9.9 vs 10.8 months; $\mathrm{HR}=1.07 ; P=0.678$ ). This is the greatest OS benefit ever seen in advanced NSCLC trials.

Grade 3 or 4 adverse events were generally low in the pemetrexed arm. Grade 3 or 4 neutropenia and fatigue 
occurred more in patients who received pemetrexed compared with patients having placebo $(P<0.05$ for both comparisons). Patients on pemetrexed had significantly greater median time to first worsening of pain compared with placebo (6.1 vs 4.6 months; HR=0.76; $P=0.041$ ); however, time to deterioration of other symptoms was not statistically different between the 2 arms.

Although $67 \%$ of patients on the placebo arm received a second-line therapy, only $19 \%$ of them received pemetrexed which may have influenced the survival outcome. Of the patients on pemetrexed maintenance, $50 \%$ received a third-line treatment later, most commonly with docetaxel or erlotinib.

\section{Ongoing trials of maintenance therapy with pemetrexed}

As mentioned above, the JMEN trial evaluated maintenance pemetrexed in patients who had not been treated with the drug during the induction phase. Hence, the doubt as to whether the observed effect results from the early use of second-line treatment. To resolve this question, the S-124 trial ramdomized patients with advanced nonsquamous NSCLC who have not progressed during the induction therapy using cisplatin plus pemetrexed to maintenance pemetrexed or placebo. The results of this trial are not expected before 2011.

Also an open-label phase 3 study is currently recruiting patients with stage $3 \mathrm{~B}$ or 4 NSCLC into a randomized trial (Eli-Lilly H3E-MC-JMHD). In 1 arm of the study, patients receive pemetrexed plus carboplatin and bevacizumab followed by maintenance pemetrexed and bevacizumab. In another arm, patients receive paclitaxel plus carboplatin and bevacizumab followed by maintenance bevacizumab.

\section{Maintenance erlotinib and gefitinib}

A recent phase 3 study (Sequential Tarceva in unresectable NSCLC; SATURN) has examined the role of EGFR-TKI, erlotinib, as a maintenance therapy in advanced NSCLC. ${ }^{18}$ Following first-line treatment with platinum-based doublet therapy, a total of 889 patients with nonprogressive disease were randomized to receive either erlotinib $(n=437)$ or placebo $(n=447)$. Maintenance erlotinib significantly improved PFS in the overall patient population $(\mathrm{HR}=0.71 ; P<0.0001)$ and in patients with EGFR-positive tumors $(\mathrm{HR}=0.69 ; 95 \%$ CI, 0.58-0.82; $P<0.0001)$ when compared with placebo. On subgroup analysis, the HR for patients with adenocarcinoma was $0.6(P \leq 0.0001)$ and for squamous histology was $0.76(P=0.015)$ with erlotinib. The HR for the 49 EGFR mutation positive patients was $0.1(P=0.0001)$, but was still significant with a HR of 0.78 in wild-type cases $(P=0.0185)$. Adverse events were higher but rarely severe in the erlotinib arm compared with placebo (rash $9 \%$ and diarrhea $2 \%$ vs 0 , respectively). An update of this trial was presented at the International Association for the Study of Lung Cancer Congress in San Francisco in $2009^{19}$ and showed a small, but statistically significant improvement in median survival (from 11.0 to 12.00 months; $P=0.008$ ). Interestingly, the HR for wild-type EGFR patients was similar to that for those with activating mutations $(0.77,0.83)$, although there were only 49 mutation positive cases.

There have also been 2 trials examining maintenance gefitinib. In the SWOG 0023 trial, ${ }^{20}$ patients with stage $3 \mathrm{~A}$ (N2) and 3B disease received induction therapy with cisplatin plus etoposide and concurrent radiotherapy. Patients with nonprogressive disease continued to receive consolidation chemotherapy with 3 cycles of docetaxel. Following this, the 243 nonprogressors were randomized to maintenance gefitinib $250 \mathrm{mg}$ /day or placebo. Rather surprisingly, there was a significant OS disadvantage to patients receiving gefitinib $(P=0.013)$. This was not due to toxicity and remains unexplained.

A Japanese phase 3 study randomized 604 chemotherapynaive patients to either receive up to 6 cycles of platinumdoublet chemotherapy or 3 cycles of chemotherapy followed by gefitinib until disease progression. ${ }^{21}$ There was no significant difference in OS, the primary end point (13.7 vs 12.9 months; $P=0.10$ ); however, median PFS was statistically significantly prolonged in the gefitinib arm $(\mathrm{HR}=0.68$; $P<0.001)$. On subset analysis, patients having maintenance gefitinib with adenocarcinoma did significantly better than patients with adenocarcinoma having just chemotherapy $(\mathrm{HR}=0.79 ; P=0.03)$.

\section{Conclusion}

Following a limited number of cycles of response, induction chemotherapy patients with advanced NSCLC are generally happy to be told that treatment can be discontinued to allow a period of freedom from the cumulative side effects and frequent hospital attendances associated with continuing treatment. Until recently, the weight of evidence supported this approach. However, 2 developments have forced a reevaluation of this question; first, the development of a novel chemotherapy agent, pemetrexed, which as a single agent, is very well tolerated and seems free from serious cumulative toxicity when given (with vitamin supplementation) intravenously for every 3 weeks; and second, the emergence of orally active EGFR-TKIs with activity in some cases of advanced 
NSCLC, which also seem to lack serious cumulative toxicity. Furthermore, above summarized evidence has suggested that the use of these agents as maintenance therapy can prolong OS when compared with placebo in patients with response or at least stable disease following induction therapy.

For erlotinib, the OS gain is modest and at present there is no clear evidence of a subgroup benefiting more than others, although the PFS extension was much greater in EGFR mutation cases. Approval for maintenance erlotinib has been declined by the US Food and Drug Administration.

For pemetrexed, there was no improvement in OS for squamous cancers but for nonsquamous disease it was 5 months, which is a considerable effect in the context of a single agent in advanced NSCLC. In addition, there is a plausible biological explanation for the differential histological effect based on tumor TS expression. In the United Kingdom, maintenance pemetrexed has been approved by National Institute for Clinical Excellence for nonsquamous advanced NSCLC patients who have responded to induction chemotherapy consisting of a platin drug plus a second agent not including pemetrexed.

The OS outcomes of these trials have been criticized since only a minority of patients in the placebo arms went on to receive the trial agent on progression. This raises the question of whether the observed effect simply results from the early administration of an active second-line therapy that was not available to some control patients who may have deteriorated rapidly and been ineligible to receive the second-line treatment. It is well known that even in trials deliberately designed to evaluate second-line therapy following a standard first-line regimen, as many as $50 \%$ or more of all cases never receive the second-line intervention. The result of the Fidias trial of "early" (or consolidation) vs late second-line docetaxel supports this possible explanation. This approach has not been widely adopted for docetaxel not only because the OS benefit did not quite reach statistical significance, but also because docetaxel is not well tolerated in less fit patients. Pemetrexed (and erlotinib) in contrast, are generally well tolerated even in patients who have had induction, cisplatin-based chemotherapy. Certainly for pemetrexed, where there seems to be a significant and substantial OS advantage for maintenance pemetrexed, at least in nonsquamous cases, the drawbacks of docetaxel do not apply and it may not matter that the observed effect results from the early administration of an active second-line agent. More intensive follow-up of patients following induction therapy would certainly reduce the proportion of patients never receiving an active second-line agent but would also, reduce the benefit of a "treatment holiday" since frequent hospital attendances and imaging would still be required. In our view, there would still be some cases whose condition would deteriorate rapidly making them ineligible physically or emotionally for second-line therapy.

The ultimate test of whether "maintenance" is more than early second line requires a trial in which the test agent is used in induction as well as in maintenance such as the Lilly S124 trial. In this, patients with nonsquamous NSCLC who responded to cisplatin plus pemetrexed first line were randomized to maintenance pemetrexed or placebo. It has completed accrual and will be reported during 2011. In the meantime, maintenance pemetrexed is only licensed for cases of nonsquamous NSCLC who have not had pemetrexed during induction. In the United Kingdom, this is a diminishing number as pemetrexed is used increasingly for induction in these patients. The future for maintenance erlotinib is problematic since the effect size was modest and increasingly those cases likely to benefit most, ie, those with EGFR mutations, will have first-line gefitinib.

\section{Disclosure}

The authors report no conflicts of interest in this work.

\section{References}

1. Jemal A, Murray T, Wand E, et al. Cancer statistics, 2005. CA Cancer J Clin. 2005;55:10-30

2. Non-small Cell Lung Cancer Collaborative Group. Chemotherapy in non-small cell lung cancer: a meta-analysis using updated data on individual patients from 52 randomised clinical trials. BMJ. 1995;311 (7010):899-909.

3. NSCLC Meta-Analyses Collaborative Group. Chemotherapy in addition to supportive care improves survival in advanced non-small-cell lung cancer: a systematic review and meta-analysis of individual patient data from 16 randomized controlled trials. J Clin Oncol. 2008;26(28): 4617-4625.

4. Delbaldo C, Michiels S, Syz N, Soria JC, Le Chevalier T, Pignon JP. Benefits of adding a drug to a single-agent or a 2-agent chemotherapy regimen in advanced non-small-cell lung cancer: a meta-analysis. JAMA. 2004;292:470-484.

5. Kelly K, Crowley J, Bunn PA Jr, et al. Randomized phase III trial of paclitaxel plus carboplatin versus vinorelbine plus cisplatin in the treatment of patients with advanced non-small-cell lung cancer: a Southwest Oncology Group trial. J Clin Oncol. 2001;19(13):3210-3218.

6. Schiller J, Harrington D, Belani C, et al. Comparison of four chemotherapy regimens for advanced non-small cell lung cancer. $N$ Engl J Med. 2002;346:92-98.

7. von Plessen C, Bergman B, Andresen O, et al. Palliative chemotherapy beyond three courses conveys no survival or consistent quality-of-life benefits in advanced non-small-cell lung cancer. Br J Cancer. 2006; 95:966-973.

8. Socinski MA, Schell MJ, Peterman A, et al. Phase III trial comparing a defined duration of therapy versus continuous therapy followed by second-line therapy in advanced-stage IIIB/IV non-small-cell lung cancer. J Clin Oncol. 2002;20:1335-1343.

9. Azzoli CG, Baker S Jr, Temin S, et al. American Society of Clinical Oncology Clinical Practice Guideline update on chemotherapy for stage IV non-small-cell lung cancer. J Clin Oncol. 2009;27(36) $6251-6266$ 
10. Zhao R, Babani S, Gao F, Liu L, Goldman ID. The mechanism of transport of the multitargeted antifolate. MTA-LY231514, and its cross-resistance pattern in cell with impaired transport of methotrexate. Clin Cancer Res. 2000;6:3687-3695.

11. Schultz RM, Patel VF, Worzalla JF, Shih CA. Role of thymidylate synthase in the antitumor activity of the multitargeted antifolate, LY231514. Anticancer Res. 1999;19:437-443.

12. Shih C, Chen VJ, Gossett LS, et al. LY231514, a pyrrolo(2,3-d) pyrimidine-based antifolate that inhibits multiple folate-requiring enzymes. Cancer Res. 1997;57:1116-2011.

13. Rollins KD, Lindley C. Pemetrexed: a multitargeted antifolate. Clin Ther. 2005;27(9):1343-1382.

14. Hanna N, Shepherd FA, Fosella FV, et al. Randomized phase III trial of pemetrexed versus docetaxel in patients with non-small-cell lung cance previously treated with chemotherapy. J Clin Onc. 2004;22: 1589-1597.

15. Scagliotti GV, Parikh P, von Pawel J, et al. Phase III study comparing cisplatin plus gemcitabine with cisplatin plus pemetrexed in chemotherapy-naive patients with advanced-stage non-small-cell lung cancer. J Clin Oncol. 2008;26:3543-3551.

16. Fidias PM, Dakhil SR, Lyss AP, et al. Phase III study of immediate compared with delayed docetaxel after front-line therapy with gemcitabine plus carboplatin in advanced non-small-cell lung cancer. J Clin Oncol. 2009;27:591-598.
17. Ciuleanu T, Brodowicz T, Zielinski C, et al. Maintenance pemetrexed plus best supportive care versus placebo plus best supportive care for nonsmall-cell lung cancer: a randomised, double-blind, phase 3 study. Lancet. 2009;374:1432-1440.

18. Cappuzzo F, Ciuleanu T, Stelmakh L, et al. SATURN: a doubleblind, randomized, phase III study of maintenance erlotinib versus placebo following nonprogression with first-line platinum-based chemotherapy in patients with advanced NSCLC. J Clin Oncol. 2009; 27 Suppl:407s.

19. Cappuzzo F, Coudert B, Wierzbicki R, et al. Efficacy and safety of erlotinib as first-line maintenance in NSCLC following non-progression with chemotherapy: results from the phase III SATURN study (abstract A282.281). J Thorac Oncol. 2009;4(suppl 1):S289.

20. Kelly K, Chansky K, Gaspar LE, et al. Phase III trial of maintenance gefitinib or placebo after concurrent chemoradiotherapy and docetaxel consolidation in inoperable stage III non-small-cell lung cancer: SWOG S0023. J Clin Oncol. 2008;26:2450-2456.

21. Takeda K, Hida T, Sato T, et al. Randomized phase III trial of platinum-doublet chemotherapy followed by gefitinib compared with continued platinum-doublet chemotherapy in Japanese patients with advanced non-small-cell lung cancer: results of a West Japan Thoracic Oncology Group Trial (WJTOG0203). J Clin Oncol. 2010;28(5): 753-760.
Lung Cancer: Targets and Therapy

\section{Publish your work in this journal}

Lung Cancer: Targets and Therapy is an international, peer-reviewed, open access journal focusing on lung cancer research, identification of therapeutic targets and the optimal use of preventative and integrated treatment interventions to achieve improved outcomes, enhanced survival and quality of life for the cancer patient. Specific topics covered in the journal include:

\section{Dovepress}

Epidemiology, detection and screening; Cellular research and biomarkers; Identification of biotargets and agents with novel mechanisms of action; Optimal clinical use of existing anticancer agents, including combination therapies; Radiation and surgery; Palliative care; Patient adherence, quality of life, satisfaction; Health economic evaluations. 